\title{
Travelers' Diarrhea in West Africa and Mexico: Fecal Transport Systems and Liquid Bismuth Subsalicylate for Self-Therapy
}

\author{
Robert Steffen, John J. Mathewson, \\ Charles D. Ericsson, Herbert L. DuPont, \\ Andrée Helminger, Timothy K. Balm, Knut Wolff, \\ and Felix Witassek
}

\author{
From the Division of Epidemiology and Prevention of \\ Communicable Disease, Institute of Social and Preventive \\ Medicine, and the Institute of Parasitology, University of \\ Zurich, Zurich, Switzerland; the Program in Infectious \\ Diseases and Clinical Microbiology, University of Texas \\ Health Science Center at Houston, Houston, Texas; and the \\ Proctor \& Gamble Company, Cincinnati, Ohio
}

\begin{abstract}
The goals of this study were threefold: to compare the etiology of travelers' diarrhea in West Africa and Mexico, to evaluate two fecal transport systems for the recovery of enteropathogens, and to verify the efficacy of liquid bismuth subsalicylate (BSS) in different locations and under different entrance criteria for disease severity. The study populations consisted of 133 European tourists in West Africa and 112 American students in Mexico who had suffered from travelers' diarrhea. In $60 \%$ and $38 \%$ of the stool samples at the two study sites, similar proportions of enteropathogens were detected. A two-vial system consisting of Enteric Plus medium and polyvinyl alcohol fixative was slightly superior for identifying enteric pathogens than was a three-vial system with buffered glycerol saline, Cary-Blair medium with campylobacter antibodies, and polyvinyl alcohol fixative. In a parallel, double-blind, randomized trial, BSS significantly shortened disease duration at both study sites.
\end{abstract}

Travelers' diarrhea is the most frequent health impairment affecting travelers to developing countries [1]. The fact that clinical symptoms are similar regardless of location suggests common etiologies. Consequently, agents used for self-therapy should show a similar efficacy in various countries.

Received for publication 22 September 1987 and in revised form 10 December 1987.

This study was conducted with the approval of institutional review boards in Switzerland and at the University of Texas.

This work was supported by grants from the Proctor \& Gamble Company.

We thank the various tour operators, tour guides, and the managements of the hotels in West Africa for their cooperation; Elisabeth Vollenweider and Barbara Nägeli for secretarial assistance; and Beth Urech-Rankin for linguistic advice. Drs. Gaudenz Bachmann, Markus Burla, Claudia Ficklocki, Rolf Heusser, Hanspeter Jung, Peter Kälin, Robert Kurmann, Bernhard Meier, Daniel Meili, Toni Reichmuth, Alois Siegwart, and Urs Wilhelm were the residents stationed in West Africa. Drs. Gilbert Oddo, Carlos Felix, Philip C. Johnson, and Francisco Javier de la Cabada and Jon Ann M. Bitsura, Margaret W. DuPont, Abraham Miranda, David Ocho, Michael McGinnis, Charlton Wilson, Laura Gutierrez, Scott Thornton, Teresa Pimienta, Ana Maria Garcia, and the students and staff of the University of San Diego and the University of Arizona Guadalajara Summer Programs participated in the Mexico portion of the study.

Please address requests for reprints to Dr. Robert Steffen, Institute of Social and Preventive Medicine, Sumatrastrasse 30, CH 8006 Zurich, Switzerland.
Previous studies, however, have sometimes demonstrated considerable differences in identified microorganisms causing travelers' diarrhea in differing locations $[2,3]$. This finding may be due to the specific populations (military, Peace Corps Volunteers, students) that were investigated and also to the different microbiological assays used. Therefore, this study was undertaken not only to compare etiologic agents in two widely different locations, but also to test two preservation kits for transportation of stool specimens from the field in West Africa to the diagnostic laboratory for microbiological evaluation. In addition, the effectiveness of bismuth subsalicylate (BSS), formerly tested in Latin America [4, 5], was to be examined not only in a distinctly different geographic location, but also under different entrance criteria for disease severity that more closely mimic actual usage patterns.

\section{Subjects and Methods}

Recruitment in West Africa. In West Africa, adult European tourists of either sex who were staying at a large hotel either in The Gambia or in Togo were asked to participate in a double-blind, parallel, randomized study. Recruitment was performed between November 1985 and February 1986 by a Swiss medical resident who informed the guests that 
if they developed diarrhea, they should collect a stool sample and report to a hotel room designated as the "diarrhea clinic." Diarrhea was defined as one or more watery (pourable) stools or as one or more pasty (does not retain its shape) stools accompanied by nausea, vomiting, or abdominal pain and cramps. Upon presentation, the resident obtained a signed informed consent and completed a medical history on each volunteer. Eligible subjects were randomly assigned to ingest either $1050 \mathrm{mg}$ of BSS liquid every $1 \mathrm{~h}$ up to a maximum of four doses per day for two days or a similar-tasting placebo. Medication was to be started immediately and to be discontinued when the symptoms subsided. Every subject was given diary cards to record the time of passage of each stool, the stool consistency, the severity of associated symptoms, side effects, and time and amount of medication taken during the $0-24-$ and $24-48$-h treatment periods.

Comparison of fecal transport systems. After collection, each stool specimen was immediately placed into two different transport systems for weekly shipment at room temperature $(\sim 23 \mathrm{C})$ via commercial airline to Houston. The content of these two systems was as follows: $(I)$ a three-vial system consisting of buffered glycerol saline (BGS), Cary-Blair medium with campylobacter antibiotics (CB), and polyvinyl alcohol (PVA) fixative (Remel, Lenexa, Kan) and (2) a two-vial system consisting of Fekal ${ }^{\circledR}$ Enteric Plus medium (modified Cary-Blair medium) and PVA fixative (Trend Scientific, Minneapolis). The PVA vials for the identification of parasitic pathogens were the same in both transport systems and were not compared. Each specimen from the Remel two-vial system and the Enteric Plus system was processed separately for bacterial enteropathogens to determine which system was the best for transporting fecal specimens. Additionally, for comparison with the PVA vial, a sample placed in merthiolate formaline (MF) was forwarded weekly to the Zurich Institute of Parasitology.

Recruitment in Mexico. In Guadalajara, Mexico, male and female adults (mostly American students from the University of Arizona [Tucson], the University of San Diego [San Diego], or the Universidad Autonoma de Guadalajara [Guadalajara]) were asked to participate in a double-blind, parallel, randomized three-day efficacy study. From February to November 1985, prospective participants were informed that if they developed diarrhea, they should report to the "diarrhea clinic." Diarrhea was defined as one or more symptoms of enteric infection, plus either three or more unformed stools in an 8-h period or four or more such stools in a 24-h period. A stool was obtained before treatment was begun, verified as unformed, and processed for enteric pathogens at an on-site laboratory.

After informed consent was obtained, each subject was randomly assigned to use either liquid BSS according to a two-day regimen of ingesting $525 \mathrm{mg}$ every 30 min up to a maximum of eight doses per day or a similar-tasting placebo. Each subject was given diary cards as in West Africa and was instructed to return to the clinic each day. The diary cards were maintained for $72 \mathrm{~h}$ : the 48 -h treatment period and the ensuing $24 \mathrm{~h}$ posttherapy.

At both study sites, all subjects who had taken other antidiarrheal or antimicrobial agents before or during the surveillance period were excluded. Also excluded were those volunteers who took $<75 \%$ of the prescribed study medications or took longer than $8 \mathrm{~h}$ to complete the total daily dose.

Statistical methods. In the BSS efficacy trial, all data entries were completed before breaking the medication code. Statistical evaluation of the data was performed using the SPSSX ${ }^{\circledR}$ (SPSS Inc., Chicago) and SAS ${ }^{\circledR}$ (SAS Institute, Cary, NC) systems. Survival analysis methods were used to compare disease duration, analysis of variance was used to compare stool frequencies, and $\chi^{2}$ tests were used to compare stool consistencies. Statistical significance was defined as a two-sided $\alpha$-risk of $\leqslant .05$. In the patients still experiencing symptoms at the end of the surveillance period, the maximum value of $48 \mathrm{~h}$ (West Africa) or $72 \mathrm{~h}$ (Mexico) was used to calculate the time until improvement or relief.

Microbiology. All stool specimens were inoculated onto MacConkey's, Tergitol-7, and xylose-lysine-desoxycholate (XLD) agars and into selenite-F enrichment broth (Difco Laboratories, Detroit). These media were incubated at $37 \mathrm{C}$ for $18 \mathrm{~h}$. The selenite$\mathrm{F}$ broth was then streaked onto a plate of XLD that was incubated at $37 \mathrm{C}$ for $18 \mathrm{~h}$ to enrich Salmonella and Shigella. Specimens were also streaked onto thiosulfate-citrate-bile-sucrose (TCBS) media (Difco) to isolate Vibrio spp. A plate of campy-blood agar and a tube of campylobacter enrichment media (Scott Laboratories, Houston) were also inoculated and incubated for $24-48 \mathrm{~h}$ at $42 \mathrm{C}$ in an atmosphere of $5 \%$ $\mathrm{O}_{2}$ to isolate Campylobacter jejuni. The enrichment medium was subcultured to another plate of campyblood agar at $24 \mathrm{~h}$. 
Non-lactose-fermenting colonies from the primary isolation plates were inoculated onto triple sugariron agar slants and lysine-iron agar slants and into sulfide-indole-motility media (Difco). Presumptive enteric pathogens were tested for oxidase production and were confirmed by using the API 20E system (Analytab Products, Plainview, NY). Salmonella and Shigella were serogrouped with commercial typing antisera (API-SerImmSure ${ }^{\circledR}$; Analytab). C. jejuni was identified by gram stain, oxidase test, catalase test, susceptibility to nalidixic acid, and resistance to cephalothin. Five Escherichia coli-like colonies were picked from each specimen and tested for the production of heat-labile and heat-stable enterotoxins (LT and ST, respectively) by using the Y-1 adrenal cell and suckling mouse assays $[6,7]$. E. coli that did not produce ST or LT were then tested for HEp-2 cell adherence [8], and adherent strains (enteroadherent $E$. coli) were further tested for somatic serogroups of enteropathogenic $E$. coli [8]. Enteroinvasive $E$. coli were detected by biotyping and testing strains that were lysine decarboxylase negative and nonmotile in the Sereny test [9] for invasiveness. Parasitic enteropathogens were sought from the PVA

Table 1. Enteropathogens identified in pretreatment stool specimens.

\begin{tabular}{lcc}
\hline & \multicolumn{2}{c}{ Study site } \\
\cline { 2 - 3 } Enteropathogen isolated & $\begin{array}{c}\text { West Africa } \\
(n=132)\end{array}$ & $\begin{array}{c}\text { Mexico } \\
(n=112)\end{array}$ \\
\hline ETEC* & $55(42)$ & $27(24)$ \\
ETEC ST & 30 & 9 \\
ETEC LT & 6 & 8 \\
ETEC ST/LT & 19 & 10 \\
Enteroadherent $E$. coli & $9(7)$ & $7(6)$ \\
Localized adherence & 3 & 1 \\
Diffuse adherence & 6 & 6 \\
Enteroinvasive $E$. coli & 0 & $2(2)$ \\
Shigella spp. & $9(7)$ & $7(6)$ \\
Salmonella spp. & $5(4)$ & $1(1)$ \\
C. jejuni & $1(1)$ & $2(2)$ \\
Cryptosporidium & $2(2)$ & 0 \\
Aeromonas spp. & 0 & $1(1)$ \\
Plesiomonas shigelloides & $7(5)$ & 0 \\
E. histolytica & $2(2)$ & 0 \\
G. lamblia & 0 & $2(2)$ \\
Specimen with pathogens & $79(60)$ & $42(38)$ \\
No agent identified & $53(40)$ & $70(62)$ \\
\hline
\end{tabular}

NOTE. Pathogens from the same patient were counted separately. One sample from West Africa was lost. Data are expressed as no. of enteropathogens identified $(\%$ of total specimens isolated).

* ETEC = enterotoxigenic E. coli.
Table 2. Comparison of the Remel and Fekal transport systems for the isolation of bacterial enteropathogens from diarrheal stool specimens.

\begin{tabular}{|c|c|c|c|c|}
\hline \multirow[b]{2}{*}{$\begin{array}{l}\text { Enteropathogen } \\
\text { isolated }\end{array}$} & \multicolumn{3}{|c|}{ Transport system used } & \multirow[b]{2}{*}{ Total } \\
\hline & $\begin{array}{l}\text { Remel } \\
\text { alone }\end{array}$ & $\begin{array}{l}\text { Fekal } \\
\text { alone }\end{array}$ & Both & \\
\hline ETEC ST* & 2 & 3 & 8 & 13 \\
\hline ETEC LT* & 0 & 3 & 3 & 6 \\
\hline ETEC ST/LT* & 1 & 1 & 4 & 6 \\
\hline EAEC & 3 & 3 & 3 & 9 \\
\hline Shigella & 3 & 0 & 6 & 9 \\
\hline Salmonella & 1 & 1 & 3 & 5 \\
\hline C. jejuni & 0 & 0 & 1 & 1 \\
\hline Plesiomonas & 1 & 6 & 0 & 7 \\
\hline Total detected & $11(20 \%)$ & $17(30 \%)$ & $28(50 \%)$ & 56 \\
\hline
\end{tabular}

NOTE. All specimens were obtained from European travelers to West Africa. ETEC = enterotoxigenic E. coli; EAEC = enteroadherent $E$. coli.

${ }^{*} E$. coli from both transport systems were tested for enterotoxins only in 66 of the 132 patients.

fixative. Two slides were prepared, air-dried, and heat fixed. One slide was stained by using the trichrome method [10] and was examined microscopically for fecal leukocytes, Entamoeba histolytica, and Giardia lamblia. The other slide was stained by using a modified Kinyoun method for Cryptosporidium [11]. The MF samples were analyzed by using the method of Blagg et al. [12], except that iodine was added to the stool sediment just before examination.

\section{Results}

In West Africa, 133 tourists were enrolled, and 130 (67 receiving BSS, 63 receiving placebo) were completely evaluated. In Mexico, 112 students were recruited, and 99 (49 receiving BSS, 50 receiving placebo) successfully completed the trial. No significant differences were found at either study site between the active medication and the placebo groups with respect to sex, age, type of malaria prophylaxis, mean length of illness, number of unformed stools passed, and other symptoms before therapy.

Microbiology and effectiveness of transport media. Table 1 shows the enteropathogens isolated from patients receiving treatment in West Africa and Mexico. Enterotoxigenic E. coli were the most common pathogens isolated in both study locations, although they were identified more frequently in West Africa ( $42 \%$ vs. $24 \%$ ). Enteroadherent $E$. coli and Shigella were the next most-common pathogens in 
both studies. $P$. shigelloides, $E$. histolytica, and Cryptosporidium were only identified in West Africa, whereas G. lamblia, enteroinvasive $E$. coli, and Aeromonas spp. were only isolated from patients in Mexico. No enteropathogenic $E$. coli serotypes were found in either study. The isolation of bacterial enteropathogens from the two transport systems is given in table 2. The Remel system was more effective in isolating Shigella and yielded three strains that were not found using the Fekal system. The Fekal system identified six $P$. shigelloides isolates, whereas the Remel system detected only ohe. Fekal was also better for isolating LT-positive $E$. coli. The rest of the results probably reflect the fact that using two specimens increases the probability of isolating enteropathogens. Overall, the same pathogen was isolated by both systems in $50 \%$ of the cases. The Remel system allowed the isolation of $70 \%$ of all the pathogens that were identified, and the Fekal system allowed the isolation of $80 \%$ of the bacterial enteropathogens.

Whereas the PVA fixative revealed two infections with $E$. histolytica, only one was found by the MF method. No other pathogenic parasites were detected with either of these two methods.

Efficacy of BSS. The time from initiation of
Figure 1. Number and proportion of stools with the indicated consistency ( $\square$, firm; soft; watery) in the BSS and in the placebo groups in West Africa (top) and Mexico (bottom). Time on the horizontal axis indicates the hours after initiation of treatment.
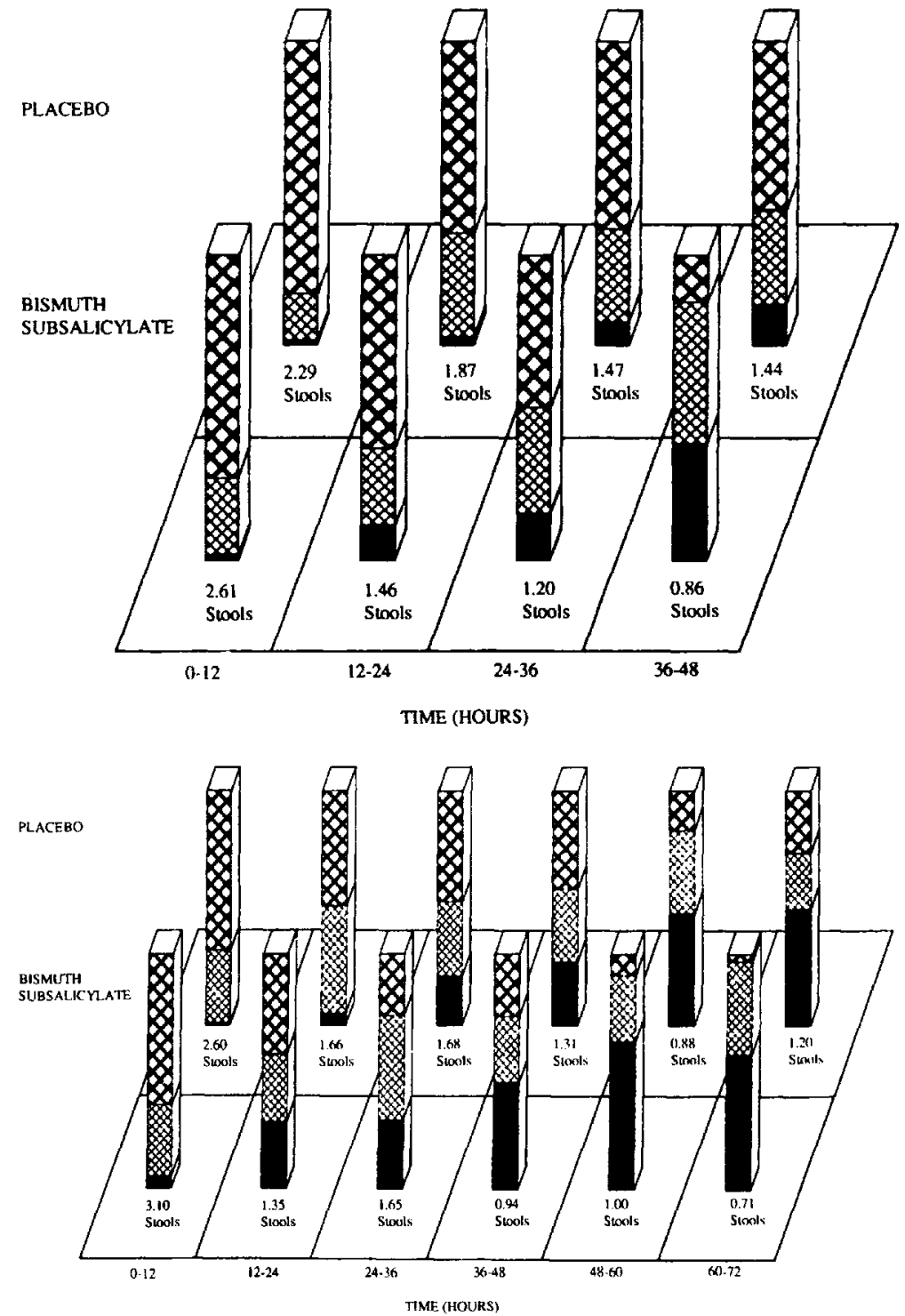
therapy to the passage of the last unformed stool was significantly diminished in West Africa ( $25.8 \mathrm{~h}$ vs. $34.5 \mathrm{~h}, P<.01)$ and in Mexico $(24.2 \mathrm{~h}$ vs. $31.4 \mathrm{~h}, P$ $=.02$ ). Also, the time to total relief of all symptoms after therapy was begun was significantly shortened by treatment with BSS in West Africa (33.1 h vs. 38.0 h, $P=.01)$, as well as in Mexico ( $36.0 \mathrm{~h}$ vs. $47.5 \mathrm{~h}$, $P<.01$ ). Figure 1 shows the decrease in unformed stools passed and the improvement in stool consistency over the period of investigation at both study locations. The active medication reduced the average number of unformed stools in the entire study period by $18 \%$ in West Africa and by $16 \%$ in Mexico. BSS relieved severe illness (i.e., seven or more bowel movements per day) as favorably as mild illness. Although the entire disease state was shortened with BSS therapy, there were insufficient numbers to observe significant differences for any of the individual concomitant symptoms, such as abdominal pain and cramps, nausea, vomiting, or chills. In West Africa, diarrhea persisting after $48 \mathrm{~h}$ of treatment was noted in $21(31 \%)$ of patients receiving BSS and in $36(57 \%)$ of patients receiving placebo $(P$ $=.01)$. In Mexico, $8(16 \%)$ of patients treated with BSS and $21(42 \%)$ receiving placebo $(P<.01)$ needed alternative therapy or had continuing diarrhea at the end of $72 \mathrm{~h}$ of observation.

In West Africa, of the patients treated with BSS, $46 \%$ stated they were completely cured at the end of the 48-h treatment period, whereas only $25 \%$ of the patients receiving placebo felt their condition had completely resolved. Similar results were obtained in Mexico, where the respective rates were $53 \%$ and $39 \%$ after $48 \mathrm{~h}$. These rates of cure were corroborated by the investigators' evaluation.

BSS was well tolerated at both study sites; no serious adverse reactions occurred. In the total study population, a black tongue was noted by $25(22 \%)$ of 116 volunteers receiving BSS and by $5(4 \%)$ of 113 receiving placebo. Black stools were reported by $79(69 \%)$ of those who used BSS and by $12(11 \%)$ of those receiving placebo. These reactions are anticipated effects of BSS therapy and were mentioned both in the informed consent and on the diary cards. The occurrence of a black tongue in individuals in the placebo group was likely a result of exaggerated concerns due to our previous warnings. Excluding such discoloration, no significant differences were observed between the active treatment and the placebo group; the number of subjects reporting tinnitus was $9(8 \%)$ vs. $4(4 \%)$, and the number report- ing constipation was $13(12 \%)$ vs. $19(18 \%)$, respectively.

\section{Discussion}

In this first etiologic assessment of travelers' diarrhea in West Africa, similar enteropathogens were found as those reported from Mexico. The only noteworthy differences were the somewhat higher proportion of enterotoxigenic $E$. coli and the occurrence of Plesiomonas in West Africa. Compared with the results of most other studies summarized at the Consensus Development Conference on Travelers' Diarrhea $[2,3]$, pathogens have been isolated in a high proportion of patients. This may partly be due to the fact that two specimens were collected to compare the two transport media. If further studies performed at other destinations in developing countries confirm the finding that the etiology of travelers' diarrhea is more or less the same everywhere, the same therapeutic regimen applies everywhere.

The use of fecal transport media to assess travelers' diarrhea has originally been described by Shore et al. [13] and refined by Morgan et al. [14]. Morgan et al. [14] provided evidence that conventional enteropathogens (enterotoxigenic $E$. coli and Shigella) were well preserved in fecal transport media for at least three weeks. In fact, more Shigella strains were recovered by using transport media than by using more timely direct plating done with the same stool specimens; BGS and modified CB (Remel) were then used. In the present study both systems worked well, but the Fekal system was slightly, but not significantly, superior. An additional advantage over the Remel system is that in the Fekal system, no extra vial of $\mathrm{CB}$ is required to isolate $C$. jejuni. Ideally, multiple culture systems would be used to optimize the isolation of bacterial pathogens. In view of the small number of $E$. histolytica isolated, no conclusions can be drawn from the comparison of PVA and MF. In a previous study [15] it was shown that formalin-fixed material yields higher numbers of $E$. histolytica cysts than does PVA. Formalin-fixed material was also clearly superior in diagnostic efficiency for $E$. coli, hookworm, and Strongyloides stercoralis. Furthermore, the merthiolate-iodine-formalin concentration method was more effective for identifying $G$. lamblia than was PVA [10]. The availability of commercial transport systems that efficiently preserve bacterial and parasitic enteropathogens for later identification means that field studies are possible in remote areas 
of the world in the absence of local microbiological facilities.

This study shows that BSS, when taken in a dose of $4.2 \mathrm{~g} / \mathrm{d}$, provides a moderate, but significant, benefit in reducing the duration of diarrheal illness, reducing the number of unformed stools, and improving the stool consistency in patients with travelers' diarrhea. This finding confirms an earlier trial [4] in which $4.2 \mathrm{~g} / \mathrm{d}$ was also shown to be effective, but even better results were obtained with a higher dosage. Furthermore, in the present study a similar beneficial effect, despite different entrance criteria for disease severity, was noted in Mexico as well as in West Africa, where the agents' efficacy had not previously been tested. In the total study population the average time until the last unformed stool was reduced by $25 \%$ at both study sites; the average time until relief from all symptoms was diminished by $14 \%$ in West Africa and by $23 \%$ in Mexico.

Taking four or eight doses of BSS within $4 \mathrm{~h}$ after beginning treatment is not convenient when starting late in the evening. Spacing out the frequency of administration might lead to more convenience as well as greater efficacy, as similarly found in prevention studies [16]. A therapeutic regimen giving BSS every $3 \mathrm{~h}$ has been successfully tested in a pediatric population [17], but this, or possibly a standard dosage regimen, needs to be confirmed in adult patients with travelers' diarrhea.

\section{References}

1. Steffen R, Boppart I. Travellers' diarrhoea. Baillière's Clinical Gastroenterology 1987;1:361-76

2. Black RE. Pathogens that cause travelers' diarrhea in Latin America and Africa. Rev Infect Dis 1986;8:S131-5

3. Taylor DN, Echeverria P. Etiology and epidemiology of travelers' diarrhea in Asia. Rev Infect Dis 1986;8:S136-41

4. DuPont HL, Sullivan P, Pickering LK, Haynes G, Ackerman PB. Symptomatic treatment of diarrhea with bismuth sub- salicylate among students attending a Mexican university. Gastroenterology 1977;73:715-8

5. Johnson PC, Ericsson CD, DuPont HL, Morgan DR, Bitsura JAM, Wood LV. Comparison of Loperamide with bismuth subsalicylate for the treatment of acute travelers' diarrhea. JAMA 1986;255:757-60

6. Donta ST, Smith DM. Stimulation of steroidogenesis in tissue culture by enterotoxigenic Escherichia coli and its neutralization by specific antiserum. Infect Immun 1974; 9:500-5

7. Dean AG, Ching Y-C, Williams RG, Harden LB. Test for Escherichia coli enterotoxin using infant mice: application in a study of diarrhea in children in Honolulu. J Infect Dis 1972;125:407-11

8. Mathcwson JJ, Johnson PC, DuPont HL, Morgan DR, Thornton SA, Wood LV, Ericsson CD. A newly recognized cause of travelers' diarrhea: enteroadherent Escherichia coli. J Infect Dis 1985;151:471-5

9. Serény B. Experimental Shigella keratoconjunctivitis. A preliminary report. Acta Microbiologica Academiae Scientarium Hungaricae 1955;2:293-6

10. Thornton SA, West AH, DuPont HL, Pickering LK. Comparison of methods for identification of Giardia lamblia. Am J Clin Pathol 1983;80:858-60

11. Ma P, Soave R. Three-step stool examination for cryptosporidiosis in 10 homosexual men with protracted watery diarrhea. J Infect Dis 1983;147:824-8

12. Blagg W, Schloegel EL, Mansour NS, Khalaf GI. A new concentration technic for the demonstration of protozoa and helminth eggs in feces. Am J Trop Med Hyg 1955;4:23-8

13. Shore EG, Dean AG, Holik KJ, Davis BR. Enterotoxinproducing Escherichia coli and diarrheal disease in adult travelers: a prospective study. J Infect Dis 1974;129:577-82

14. Morgan DR, Johnson PC, West AH, Wood LV, Ericsson CD, DuPont HL. Isolation of enteric pathogens from patients with travelers' diarrhea using fecal transport media. FEMS Lett 1984;23:59-63

15. Carroll MJ, Cook J, Turner JA. Comparison of polyvinyl alcohol- and formalin-preserved fecal specimens in the formalin-ether sedimentation technique for parasitological examination. J Clin Microbiol 1983;18:1070-2

16. DuPont HL, Ericsson CD, Johnson PC, Bitsura JAM, DuPont MW, Javier de la Cabada F. Prevention of travelers' diarrhea by the tablet formulation of bismuth subsalicylate. JAMA 1987;257:1347-50

17. Gryboski JD, Hillemeier AC, Grill B, Kocoshis S. Bismuth subsalicylate in the treatment of chronic diarrhea of childhood. Am J Gastroenterol 1985;80:871-6 